\title{
Survival in equilibrium step fluctuations
}

\author{
C. Dasgupta, ${ }^{1, *}$ M. Constantin, ${ }^{1,2}$ S. Das Sarma, ${ }^{1}$ and Satya N. Majumdar ${ }^{3}$ \\ 1 Condensed Matter Theory Center, Department of Physics, \\ University of Maryland, College Park, Maryland 20742-4111 \\ ${ }^{2}$ Materials Research Science and Engineering Center, Department of Physics, \\ University of Maryland, College Park, MD 20742-4111 \\ ${ }^{3}$ Laboratoire de Physique Quantique, UMR C5626, \\ Universite Paul Sabatier, 31062 Toulouse Cedex, France
}

\begin{abstract}
We report the results of analytic and numerical investigations of the time scale of survival or non-zero-crossing probability $S(t)$ in equilibrium step fluctuations described by Langevin equations appropriate for attachment/detachment and edge-diffusion limited kinetics. An exact relation between long-time behaviors of the survival probability and the autocorrelation function is established and numerically verified. $S(t)$ is shown to exhibit simple scaling behavior as a function of system size and sampling time. Our theoretical results are in agreement with those obtained from an analysis of experimental dynamical STM data on step fluctuations on $\mathrm{Al} / \mathrm{Si}(111)$ and $\mathrm{Ag}(111)$ surfaces.

PACS numbers: 68.35.Ja, 68.37.Ef, 05.20.-y, 0.5.40.-a
\end{abstract}

There has been much recent theoretical [1, 2] and experimental 2, 3] interest in the persistence behavior of fluctuating steps on a vicinal surface. A persistence probability $P(t)$, defined as the probability that the position ("height") of the step edge at a point along a fluctuating step does not return to its initial value (at time $t=0$ ) over time $t$ is found [1, 2, 3] in these studies to decay in time as a power-law, $P(t) \propto t^{-\theta}$, for large $t$, where $\theta$ is the so-called persistence exponent. Similar power-law behavior of the persistence probability has also been found in experiments [4] for other physical processes. It turns out that the precise definition of $P(t)$ is absolutely crucial for the power-law behavior discussed in the recent surface fluctuations literature. If, instead of considering the probability of not returning to the initial position, one defines a survival probability, $S(t)$, as the probability of the dynamical step height (at a fixed but arbitrary spatial location) not returning in time $t$ to its average ("equilibrium") level, then, quite surprisingly, it was found in a recent experimental study [2] of thermal fluctuations of surface steps that $S(t)$ actually manifests, in sharp contrast to the power-law behavior of $P(t)$, an exponential decay, $S(t) \propto \exp \left(-t / \tau_{s}\right)$, at long times, where $\tau_{s}$ is the survival time scale. This exponential behavior of $S(t)$ has remained theoretically unexplained.

In this report we provide a definitive theoretical explanation for this exponential temporal behavior of the surface fluctuation survival probability using rigorous (analytical) arguments and direct (numerical) simulations. Survival and persistence turn out to be identical in problems related to Ising spin dynamics, where one is interested in the probability that a spin has not changed its sign (has not "flipped") up to time $t[\underline{5}$. This is due to the discrete nature of Ising spin dynamics, where a spin flip ensures a change of sign with respect to both the initial and the average (or any other reference) value of the stochastic variable. In contrast, the continuous nature of surface fluctuation dynamics, where the step height is a continuous variable, leads to a fundamental qualitative difference between $P(t)$ and $S(t)$. This qualitative difference was noted as an experimental fact and an unsolved puzzle in Ref. 2] for equilibrium step fluctuations on $\mathrm{Al} / \mathrm{Si}(111)$ [6]. We emphasize that the probabilities $P(t)$ and $S(t)$ provide completely different physical information about surface step fluctuations: while persistence characterizes the universality class of the dynamical process through the persistence exponent $\theta$, survival, as discussed in this paper, provides useful information about the physical mechanisms (and their characteristic time scales) underlying step fluctuations in the long-time limit.

Another time scale that invariably enters experimental and numerical measurements of any statistical quantity is the sampling time $\delta t$ (the interval between successive measurements of the step position). An understanding of the effects 7] of a finite $\delta t$ on the measured survival probabilities is necessary for comparing experimental and numerical results with theoretical predictions.

In this paper, we present the results of a detailed study of the behavior of $S(t)$ for two linear Langevin equations that describe [ [8, 9] step fluctuations under attachment/detachment ("high-temperature") and edgediffusion ("low temperature") limited kinetics. We first show analytically that if the equilibrium autocorrelation function $C(t)$ of height fluctuations decays exponentially at long times, then $S(t)$ must also decay exponentially with a time scale that is proportional to the correlation time (the time scale of the decay of $C(t)$ ). This prediction is verified from numerical simulations of the Langevin equations. The simulation results also provide information about the dependence of the measured survival probability on the sampling time $\delta t$. We show that the survival probability $S(t, L, \delta t)$ exhibits simple scaling behavior in both models. Finally, we use available exper- 
imental data [2, 3, 10] to calculate $C(t)$ and $S(t)$ for two physical systems that are believed to be described by these two Langevin equations and show that the experimental results are consistent with our predictions.

High-temperature step fluctuations dominated by atomistic attachment and detachment at the step edge are known [8, 9] to be well described by the second-order non-conserved linear Langevin equation

$$
\frac{\partial h(x, t)}{\partial t}=\frac{\Gamma_{a} \tilde{\beta}}{k_{B} T} \frac{\partial^{2} h(x, t)}{\partial x^{2}}+\eta(x, t) .
$$

Here, $h(x, t)$ is the dynamical height fluctuation (position of the step edge measured from its equilibrium value) at lateral point $x$ along the step and time $t$, $\Gamma_{a}$ is the "step mobility", $\tilde{\beta}$ is the step-edge stiffness, and $\eta(x, t)$ is a nonconserved Gaussian noise satisfying $\left\langle\eta(x, t) \eta\left(x^{\prime}, t^{\prime}\right)\right\rangle=2 \Gamma_{a} \delta\left(x-x^{\prime}\right) \delta\left(t-t^{\prime}\right)$. Low-temperature step fluctuations dominated by the step edge diffusion mechanism are, on the other hand, described by the fourth order conserved Langevin equation

$$
\frac{\partial h(x, t)}{\partial t}=-\frac{\Gamma_{h} \tilde{\beta}}{k_{B} T} \frac{\partial^{4} h(x, t)}{\partial x^{4}}+\eta_{c}(x, t),
$$

with $\left\langle\eta_{c}(x, t) \eta_{c}\left(x^{\prime}, t^{\prime}\right)\right\rangle=-2 \Gamma_{h} \nabla_{x}^{2} \delta\left(x-x^{\prime}\right) \delta\left(t-t^{\prime}\right)$.

Space- and time-dependent correlation functions of height fluctuations in these two linear equations may be calculated [8, 9] easily by Fourier transforms. We assume that height fluctuations are measured from the spatial average of $h(x, t)$, so that the $k=0$ Fourier component of $h(x, t)$ is zero at all times. The autocorrelation function of $h(k, t)$, the Fourier transform of $h(x, t)$, has the following form in the long-time equilibrium state:

$$
\left\langle\tilde{h}\left(k, t_{1}\right) \tilde{h}\left(-k, t_{2}\right)\right\rangle=\frac{k_{B} T}{\tilde{\beta} k^{2}} \exp \left(-\Gamma \tilde{\beta} k^{z}\left|t_{1}-t_{2}\right| / k_{B} T\right),
$$

where $z=2, \Gamma=\Gamma_{a}$ for Eq.(1), and $z=4, \Gamma=\Gamma_{h}$ for Eq.(2). The autocorrelation function of height fluctuations at equilibrium is then given by

$$
\begin{aligned}
C(t) & =\left\langle h\left(x, t_{1}\right) h\left(x, t_{2}\right)\right\rangle \\
& =\frac{2 k_{B} T}{\tilde{\beta}} \int_{k_{\text {min }}}^{\infty} \frac{d k}{2 \pi} \frac{\exp \left(-\Gamma \tilde{\beta} k^{z}|t| / k_{B} T\right)}{k^{2}}
\end{aligned}
$$

where $t=t_{1}-t_{2}$ and $k_{\min }=2 \pi / L$ for a finite system of linear dimension $L$. This implies that $C(t)$ exhibits an exponential decay at long times, $C(t) \propto \exp \left(-t / \tau_{c}\right)$, where the correlation time $\tau_{c}$ is equal to $k_{B} T L^{z} /(2 \pi)^{z} \Gamma \tilde{\beta}$.

There are other physical mechanisms that may lead to an exponential decay of $C(t)$ at long times. The fluctuations of a particular step are affected by its interaction with neighboring steps. These interaction effects, which are negligible at relatively short time scales if the spacing between neighboring steps is large, may become important at long times. If one assumes that the step fluctuates in a harmonic confining potential [8], $(\lambda / 2) \int h^{2}(x) d x$, then one obtains an additional term, $-\lambda \Gamma_{a} h(x, t) / k_{B} T$, in the right-hand of Eq.(11), and $\lambda \Gamma_{h} \nabla_{x}^{2} h(x, t) / k_{B} T$ in Eq.(2). The function $C(t)$ is then given by

$$
C(t)=2 k_{B} T \int_{k_{\text {min }}}^{\infty} \frac{d k}{2 \pi} \frac{\left.\exp \left[-\Gamma\left(\tilde{\beta} k^{z}+\lambda k^{z-2}\right)|t| / k_{B} T\right)\right]}{\tilde{\beta} k^{2}+\lambda} .
$$

This, again, leads to an exponential decay of $C(t)$ at long times, with the correlation time $\tau_{c}$ a function of $\lambda$ and $L$.

There exists a rigorous theorem [11] that states that if the autocorrelation function $C(t)$ of a stationary Gaussian process decays exponentially in time, then its survival probability $S(t)$ must also decay exponentially for large $t, S(t) \propto \exp \left(-t / \tau_{s}\right)$, with the survival time scale $\tau_{s}$ proportional to the correlation time $\tau_{c}$. The constant of proportionality, $c \equiv \tau_{s} / \tau_{c}$, which must be less than unity and independent of the system size $L$, is usually nontrivial, being determined by the full functional form of $C(t)$. Since the height fluctuations in our models represent a stationary Gaussian process at equilibrium, this rigorous result applies for the survival probability of these fluctuations. Thus, we arrive at a very general, exact result that the survival probability of equilibrium step fluctuations should decay exponentially at long times if the autocorrelation function does so. This is the first important result of this article. Further, measurements of the ratio $c$ of the two time scales may provide valuable information about the nature of the processes involved in the step fluctuations.

We have investigated these aspects in a detailed numerical study in which a simple Euler scheme [1] is used to numerically integrate spatially discretized versions of Eqs.(11) and (2). All the results reported here were obtained in the equilibrium regime. $S(t)$ is measured as the probability that the height fluctuation $h_{i}$ at a particular site $i$ does not cross the average step height (which is conveniently chosen as the "zero" of the height stochastic variable) over time $t$, averaged over all sites and many $\left(\sim 10^{4}-10^{6}\right)$ independent runs. $C(t)$ is calculated exactly using discretized versions of Eqs.(4) and (5).

Typical results for $C(t)$ and $S(t)$ are shown in Fig 1 (a-c) for Eq. (11) and in Fig 2 (a-c) for Eq. (2) $[\lambda=0$ in both cases]. As indicated in the figures, we used several different values of the sampling time $\delta t$ in the measurement of $S(t)[C(t)$ is, of course, independent of $\delta t]$. It is clear from the plots that both $C(t)$ and $S(t)$ decay exponentially at long times. The time scales $\tau_{c}$ and $\tau_{s}$ are extracted from exponential fits shown in the semi-log plots as dashed straight lines. The dependence of $\tau_{c}$ on $L$ is given exactly by $\tau_{c}(L)=(L / 2 \pi)^{z}$. The results for different values of $L$ shown in Figs. 1 1 and 2 indicate that $\tau_{s}$ also increases rapidly as $L$ is increased. However, we find that the calculated values of $\tau_{s}$ extracted from the $S(t)$ data obtained for different $L$ using the same sampling time $\delta t$ exhibit small but clear deviations from the 

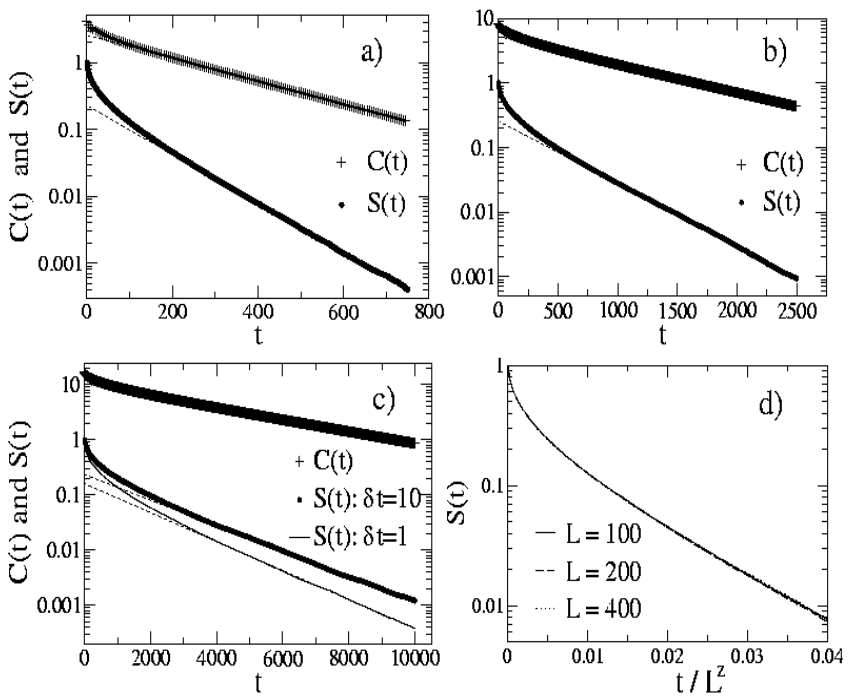

FIG. 1: $\quad S(t)$ and $C(t)$ for the Langevin equation of Eq.(1). The dashed lines are fits of the long-time data to an exponential form. In panels (a-c), the uppermost plots show the data for $C(t)$. Panel (a): $L=100, \delta t=0.625$. Panel (b): $L=200, \delta t=2.5$. Panel (c): $L=400, \delta t=10.0$ (upper plot) and $\delta t=1.0$ (lower plot). Panel (d): Finite-size scaling of $S(t, L, \delta t)$. Results for $S$ for 3 different sample sizes with the same value of $\delta t / L^{z}(z=2)$ are plotted versus $t / L^{z}$.

expected proportionality to $L^{z}$. These small deviations result from a weak dependence of $S(t)$ on the sampling time $\delta t$. As shown in Fig 1 and Fig 2b, the rate of the exponential decay of $S(t)$ at large $t$ depends weakly on the value of $\delta t$ used in the measurement of $S$. This is in accordance with the analytic predictions of Ref. [7]. Since the only time scale in the problem is $\tau_{c}$ (as mentioned above, $\tau_{s}$ should be proportional to $\tau_{c}$ ), the dependence of $S(t)$ on the sampling time $\delta t$ should involve the scaling combination $\delta t / \tau_{c}$. Since $\tau_{c}(L) \propto L^{z}$ in our models, this argument suggests that the sampling time should be chosen to be proportional to $L^{z}$ if the survival probabilities for different values of $L$ are to be tested for scaling. Indeed, as shown in Fig [1, the values of $S(t)$ obtained for $L=100,200$ and 400 using $\delta t=0.625,2.5$ and 10.0, respectively, (so that $\delta t \propto L^{z}$ with $z=2$ ) all fall on the same scaling curve when plotted as functions of $t / L^{z}$ with $z=2$. As shown in Fig 2 $\mathrm{d}$, a similar scaling collapse is obtained for Eq.(2). Here, the sampling times for different $L$ are chosen to be proportional to $L^{4}$, and the best scaling collapse is obtained when the data for different $L$ are plotted against $t / L^{z}$ with $z \simeq 3.95$. These results establish that the full function $S(t, L, \delta t)$ (not just the asymptotic long-time part) has the scaling form

$$
S(t, L, \delta t)=f\left(t / L^{z}, \delta t / L^{z}\right),
$$

where the function $f(x, y)$ decays exponentially for large values of $x$ and the rate of this decay increases slowly as $y$ is decreased. This finite-size scaling behavior of $S$, which
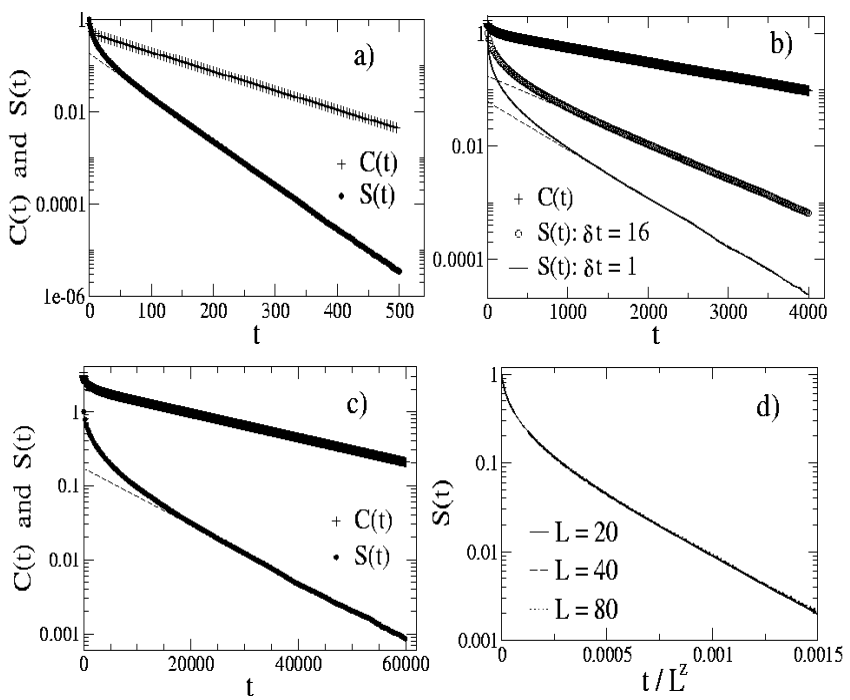

FIG. 2: $S(t)$ and $C(t)$ for the Langevin equation of Eq.(2). In panels (a-c), the uppermost plots show the results for $C(t)$. The dashed lines are fits of the long-time data to an exponential form. Panel (a): $L=20, \delta t=1$. Panel (b): $L=40$, $\delta t=16$ (upper plot) and $L=40, \delta t=1$ (lower plot). Panel (c): $L=80, \delta t=256$. Panel (d): Finite-size scaling of $S(t, L, \delta t)$. Results for $S$ for 3 different sample sizes with the same value of $\delta t / L^{z}(z=4)$ are plotted versus $t / L^{z}$.

represents the second important result of our study, is similar to that found [12] for the persistence probability in a coarsening system. However, the dependence on the sampling time, essential in our scaling considerations, was not analyzed in Ref. [12].

We have also studied the behavior of $C(t)$ and $S(t)$ for Eq. (11) when the value of $\tau_{c}$ is primarily determined by the presence of a nonzero $\lambda$ associated with step-step interaction (cf. Eq. (5) above). By varying $\lambda$ and $\delta t$ for a system with $L=400$, we find that $S(t, \lambda, \delta t)$ exhibits excellent scaling behavior as a function of $t / \tau_{c}$ if the quantity $\delta t / \tau_{c}$ is held constant. Therefore, we conclude that $S$ is a function of the scaling variables $t / \tau_{c}$ and $\delta t / \tau_{c}$, irrespective of the origin of the finite value of the correlation time $\tau_{c}$.

For $\lambda=0$, the ratio $c=\tau_{s} / \tau_{c}$ for Eq. (11) decreases from about 0.57 to about 0.41 as the ratio $\delta t / \tau_{c}$ is decreased from 0.025 to $2.5 \times 10^{-4}$, indicating that $c \simeq 0.4$ in the $\delta t \rightarrow 0$ limit. For relatively large $L$ and nonzero $\lambda$ where $\tau_{c}$ is determined primarily by the value of $\lambda$, we find that $c \simeq 0.47$ for $\delta t / \tau_{c}=0.025$. The difference between the values of $c$ for the same value of $\delta t / \tau_{c}$ in the two cases reflects the expected dependence of $c$ on the details of $C(t)$. For Eq. (2) with $\lambda=0$, the value of $c$ decreases from about 0.44 to about 0.30 as $\delta t / \tau_{c}$ is decreased from 0.01 to $6 \times 10^{-4}$. The qualitative behavior of $c$ as a function of $\delta t / \tau_{c}$ is similar in all the cases we have considered, and is consistent with the general predictions of Ref. []].

We have also used dynamical STM data to calculate 


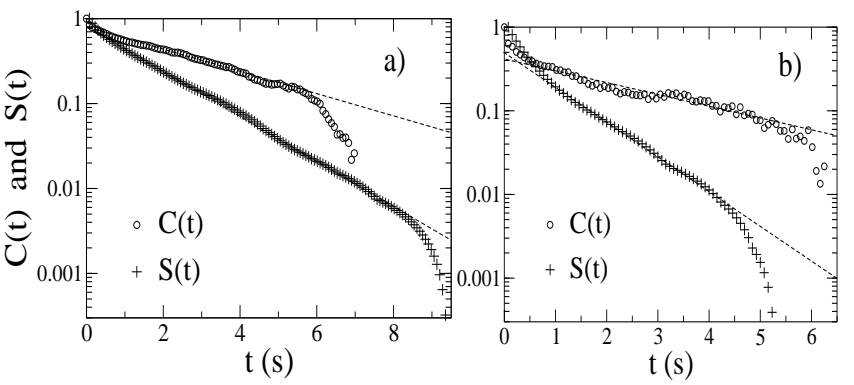

FIG. 3: $\quad S(t)$ and $C(t)$ for two experimental systems. The dashed lines are fits of the long-time data to an exponential form. Panel (a): $\mathrm{Al} / \mathrm{Si}(111)$ at $T=970 \mathrm{~K}$. Panel (b): $\mathrm{Ag}(111)$ at $T=450 \mathrm{~K}$.

$C(t)$ and $S(t)$ for two experimental systems: $\mathrm{Al} / \mathrm{Si}(111)$ at relatively high temperatures, which is believed 2, 13, 14] to provide a physical realization of Eq.(11), and $\mathrm{Ag}(111)$ at relatively low temperatures where the step fluctuations are expected [3, 10] to be governed by the conserved Eq.(21). Some of the results of this analysis are shown in $\mathrm{Fig} 3$ For $\mathrm{Al} / \mathrm{Si}(111)$ at $970 \mathrm{~K}$ we find exponential decay of both $C(t)$ and $S(t)$. The value of the ratio $c$ obtained from the estimates of $\tau_{c}$ and $\tau_{s}$ is close to 0.5 . This value is right in the middle of the range of values of $c$ obtained from our numerical study of Eq.(11). The $\mathrm{Ag}(111)$ at $450 \mathrm{~K}$ data is characterized by $c \simeq 0.34$, which is again in the range of values obtained in the numerical study of Eq.(2). We, therefore, conclude that the available experimental data on $S(t)$ and $C(t)$ are consistent with our theoretical results.

Experimental data on the $\mathrm{Al} / \mathrm{Si}(111)$ system are available at several temperatures between $770 \mathrm{~K}$ and $1020 \mathrm{~K}$. As reported in Ref. 2], $S(t)$ decays exponentially at long times at all these temperatures, with $\tau_{s}$ decreasing from $3.6 \mathrm{~s}$ to $0.9 \mathrm{~s}$ as $T$ is increased from $770 \mathrm{~K}$ to $1020 \mathrm{~K}$. Using these values of $\tau_{s}$ (actually, the corresponding values of $\tau_{c}$ obtained from the relation $\tau_{s} / \tau_{c} \simeq 0.5$ ) together with the values of the parameters $\Gamma$ and $\tilde{\beta}$ obtained from other measurements 13, 14], we have calculated an "effective length" $L_{\text {eff }}$ that would lead to the observed finite value of $\tau_{c}$ if it resulted from a finite length of the sample. The value of $L_{e f f}$ is found to decrease from $4020 \AA$ to $389 \AA$ as $T$ is decreased from $1020 \mathrm{~K}$ to $770 \mathrm{~K}$. These values are much smaller than the nominal step lengths in the experimental sample. The observed $T$-dependence of $L_{e f f}$ is inconsistent with the possibility that the finite values of $\tau_{c}$ are due to a nonzero value of the parameter $\lambda$ : the length scale associated with $\lambda$ should increase [8] as $T$ is decreased. It is possible that $L_{e f f}$ is a measure of the typical length of a step edge between adjacent points that are held fixed by some kind of pinning centers. Since pinning becomes more effective at low $T$, this mechanism would provide a qualitative explanation of why $L_{\text {eff }}$ decreases as $T$ is reduced. Yet another possibility is that
$L_{e f f}$ is a measure of the length scale over which step edge fluctuations are effectively equilibrated.

To conclude, we have shown analytically and numerically that the survival probability of equilibrium step fluctuations on vicinal surfaces decays exponentially at long times, and have established a relation between the time scales characterizing the exponential decay of the survival probability and the autocorrelation function. Our theory explains the puzzling experimental finding of an exponential decay of $S(t)$ reported in Ref. 2]. We have also shown that the survival probability exhibits simple scaling as a function of the system size and the sampling time, which plays a very important role in the measurement of $S(t)$.

The authors gratefully acknowledge discussions with E.D. Williams, O. Bondarchuk and D.B. Dougherty. S.N.M. thanks A. Bray for useful discussions. This work is partially supported by US-ONR and NSF-DMRMRSEC at the University of Maryland.

* Permanent address: Department of Physics, Indian Institute of Science, Bangalore 560012, India

[1] J. Krug et al., Phys. Rev. E 56, 2702 (1997).

[2] D. B. Dougherty et al., Phys. Rev. Lett 89, 136102 (2002).

[3] D. B. Dougherty et al., Surf. Sci. 527 L213 (2003).

[4] M. Marcos-Martin et al., Physica A 214, 396 (1995); W. Y. Tam et al., Phys. Rev. Lett. 78, 1588 (1997); B. Yurke et al., Phys. Rev. E 56, R40 (1997); G. P. Wong et al., Phys. Rev. Lett. 864156 (2001); J. Merikoski et al., Phys. Rev. Lett. 90024501 (2003).

[5] Unfortunately, rather confusing terminology has been used in the spin dynamics literature to describe this probability, including persistence, survival, and non-zerocrossing probability. Further confusion is caused by the fact that even in the surface dynamics problem, persistence and survival become the same if one starts from the initial condition of a "flat surface" (which cannot be achieved experimentally) and defines "survival" with respect to the average surface which is also "flat".

[6] We point out in this context that in Ref. [2] both of these probabilities were unfortunately called persistence probability (with respect to the average position and the initial position, respectively) causing perhaps some confusion.

[7] S. N. Majumdar et al., Phys. Rev. E 64, 015101(R) (2001); G. C. M. A. Ehrhardt et al., Phys. Rev. E 65, 041102 (2002).

[8] N. C. Bartelt et al., Surf. Sci. 273, 252 (1992).

[9] A. -L. Barabasi and H. E. Stanley, Fractal Concepts in Surface Growth (Cambridge, New York, 1995).

[10] O. Bondarchuk et al., private communication.

[11] G. F. Newell and M. Roseblatt, Ann. Math. Stat. 33, 1306 (1962).

[12] G. Manoj and P. Ray, Phys. Rev. E 62, 07755 (2000).

[13] I. Lyubinetsky et al., Phys. Rev. B 66, 085327 (2002).

[14] I. Lyubinetsky et al., Surf. Sci. 492, L671 (2001). 